\title{
Research on the Strategy of improving the ability of disaster Prevention and Mitigation from the Perspective of the overall National Security concept
}

Shuhong He, Mengfei Jiang

Faculty of Economics, Yunnan University

Kunming 650500, China

jerome112233@163.com

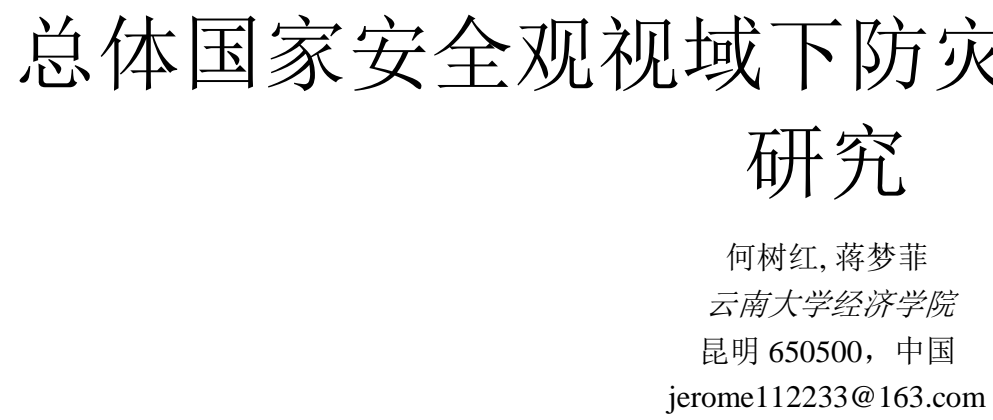

\begin{abstract}
In recent years, the global disaster problem has been deteriorating, including not only the frequent occurrence of natural disasters, but also the outbreak of various manmade disasters. Disasters have become a major factor that threatens human security and affects social development. Disasters not only cause economic losses to the state and individuals, but also easily lead to the loss of control of the normal order of society and have an important impact on the development of the country and the region. Therefore, it is necessary to raise the capacity of comprehensive disaster prevention and mitigation to the national security strategy. The overall national security concept is the general guidance of China's current national security work and has very important practical significance. Therefore, from the perspective of the overall national security concept, this paper analyzes the problems existing in the traditional disaster prevention and mitigation system, and puts forward the strategies for disaster prevention and mitigation capacity improvement and related suggestions based on the actual situation in China.
\end{abstract}

Keywords - overall national security concept; disaster prevention and mitigation; strategy research

摘要一一近年来, 全球灾害问题在不断恶化, 其中不仅包 括自然灾害的频繁发生, 还有各种人为灾害的爆发, 灾害已 经成为危及人类安全和影响社会发展的重大因素。灾害不仅 给国家和个人造成经济上的损失，还容易导致社会正常秩序 的失控, 对国家和区域的发展产生重要的影响。因此, 有必 要将综合防灾减灾能力建设上升到国家安全战略高度。总体 国家安全观是我国目前国家安全工作的总指导，具有非常重 要的现实意义。因此, 本文从总体国家安全观的角度出发, 对传统防灾减灾体系存在问题进行分析, 结合我国实际情 况, 提出防灾减灾能力提升策略及相关建议。

关键词一一总体国家安全观；防灾减灾；策略研究

\section{I. 引言}

中国是灾害频发国家, 特别是随着我国社会经济快速 发展, 我国面临的各种来自国内和国外的灾害和风险也 日益增多, 灾害类型日益复杂, 这非常不利于我国经济 社会发展。在传统灾害治理体系难以防治更加复杂的灾 害风险的情况下，如何构建综合性灾害治理体系已成为 了备受关注的课题。

\section{A. 总体国家安全观}

十八大以来，党中央结合我国实际情况和我国发展目 标, 将国家安全工作提升到新的战略高度。“总体国家 安全观” 作为新国家安全理念, 受到各学者的高度关 注, 其相关内容、意义、实践路径被广泛研究和探讨 [1]。陈秋菊（2018）提出中国 “总体国家安全观” 是我 国安全治理体系的 “顶层设计”，兼顾对内国家安全和 对外国家安全, 对我国安全治理模式和应急管理体系都 产生重要影响[2]。夏文贵（2017）也提出总体国家安全 观的提出使国家安全的内涵更加丰富, 几乎包含了全部 风险要素[3]。

\section{B. 传统防灾减灾模式}

在我国防灾减灾模式不断演进过程中，学者们对传统 防灾减灾思路进行了探讨, 陶鹏, 童星（2013）提出传 统的防灾减灾经历了从工程和技术为主的结构性防灾减 灾模式到以规划和宣传为主的非结构性防灾减灾模式再 到结构性和非结构性相结合的复合型防灾减灾模式的演 变[4]。李永祥 (2011) 提出我国复合型防灾减灾模式虽 然在一定程度上具有 “综合性防灾减灾” 特点, 但是仍 然局限于海啸、地震等自然灾害, 难以应付日益复杂的 灾害类型[5]。史培军 (2014) 提出传统防灾减灾模式具 有不注重灾前防御、灾种单一、防灾减灾体制分离等缺 陷[6]。林毓铭（2015）也提出传统防灾减灾体系中灾害 
治理对象主要是自然灾害, “灾害” 的内涵较为狭义, 没有考虑到恐怖袭击、经济危机等灾害 [7]。张海波 （2016）提出传统防灾减灾体系中不同类别灾害如自然 灾害和人为灾害之间的研究治理存在明显界限, 忽视了 各灾害之间的风险关联性和串发性，使防灾减灾效率低 下［8］。周利敏（2013）提出严重灾害的发生常常伴 随着哄抢、群众心理恐慌等情况, 这些事件超出了传统 灾害治理体系的范围, 在防灾减灾过程中如果不采取应 对措施，将会严重影响防灾减灾的效果［9］。马宝成 （2019）提到对传统防灾减灾模式进行改革必须要在总 体国家安全观下, 系统性增强国家灾害治理能力[10]。 刘杰,龚维斌（2018）提出传统防灾减灾模式将人为灾害 和自然灾害割裂开，但随着全球灾害产生机理、影响深 度广度更加复杂化, 对灾害仅仅进行局部治理难以达到 预期效果, 急切需要建立全面综合治理体系[11]。史培 军, 李宁, 叶谦 (2009) 等提出传统防灾减灾模式主体 主要是政府, 企业和个人投入较少, 不能有效发挥市场 机制的作用，因此要提高个人和企业的防灾减灾责任意 识，构建全社会参与的全面防灾减灾治理体系 [12]。

\section{C. 总体国家安全观视域下防灾减灾思路}

1942 年美国学者怀特首次将防灾减灾问题和国家 安全问题相结合进行探索, 这也是最早对于非传统国家 安全问题的研究。随着国内外学者们对国家安全问题的 研究不断深入, 防灾减灾问题不再只属于自然科学的研 究范畴, 而逐步向社会学研究范畴延伸。

我国总体国家安全观被提出后，国家安全不仅指国土 安全等传统国家安全, 还包含自然灾害, 社会灾害的治 理等方面, 引发国内学者从总体国家安全观出发对防灾 减灾问题进行研究。郭强提出当前自然灾害、人为灾害 甚至军事冲突等各类灾害并发的概率越来越高, 要实现 各类风险全面治理必须建立在总体国家安全观下，不能 只注重单一灾害的防灾减灾[13]。张海波（2016）提出 总体国家安全观具有全面性和统筹性的特点, 有助于 “多维度、全阶段、多主体”构建灾害治理框架[14]。

\section{II. 传统防灾减灾模式存在的问题}

虽然传统防灾减灾模式在演进过程中不断改善并取得 了一定的成果, 但是由于其传统思维的束缚, 并缺乏对 灾害的系统考虑和综合性防灾减灾政策设计, 它在防灾 减灾实践中暴露出防灾减灾体制分割、防灾减灾投入失 衡、不注重提高社会公众防灾减灾意识等缺陷。

\section{A. 防灾减灾体制分割}

以往的灾害研究中, 对不同灾害的研究相互独立, 灾害治理被认为是自然科学的研究领域, 很少有学者从 社会科学的角度对其探讨。在治理灾害的过程中，也是 各灾种分部门治理，分阶段治理，没有综合防灾减灾的 意识。然而在实践中, 虽然不同灾害产生机制和应对措 施各不相同, 但是它们的并发性和交叉性日益明显, 影 响的深度和广度也在增加, 这种不同灾种分开治理、分 环节治理的现状对建立综合防灾减灾体系产生非常不利 的影响。因此，有效的防灾减灾模式必须要有整体风险 意识, 统一的防灾减灾行动, 要从工程技术为主的结构 性治理模式过渡到非结构性综合治理模式, 最后从实际 情况出发，制定具有针对性的防灾减灾措施。

\section{B． 不同灾种管理体制分割}

当前的防灾减灾体系下，不同的灾害受到不同部门 的管理, 如台风由气象部门管理、水灾由水利部门管理 等[15]，这种灾害管理体制在一定程度上具有分工明确 的优点, 但是会造成部门之间不能有效沟通合作, 因而 产生灾害治理中政策的相互制约或者重叠，大大降低了 防灾减灾资源配置的效率, 甚至对防灾减灾效果产生负 面影响。

\section{C. 防灾减灾投入失衡}

防灾减灾投入失衡体现在: 第一，防灾减灾主体投入 比重失衡、即企业和个人投入占比过小 [16], 不能有效 发挥市场机制的作用; 第二, 防灾减灾投入环节失衡, 将防灾减灾的重心放在灾后重建阶段, 而忽略了前期灾 害的防范; 第三是投入和产出失衡, 只注重防灾减灾项 目的建立, 而忽略项目实行的作用和防灾减灾效果的综 合评估，使防灾减灾效率低下，甚至造成灾情恶化。

\section{D. 重技术轻理念}

以往我们对灾害的治理主要在技术层面, 只注重如何 通过技术手段来防灾减灾, 而忽略了灾害治理的制度建 设, 缺少系统性、全面性的统筹和综合防灾减灾观念, 这将导致了社会灾害防范意识薄弱。防灾减灾教育、先 进的防灾减灾理念、完善的防灾减灾制度都是从宏观层 面提高防灾减灾能力过程中必不可少的内容, 没有先进 的灾害治理理念, 就会影响防灾减灾制度的合理性, 从 而影响防灾减灾措施在实践过程中的效果。

\section{E. 公众防灾减灾意识薄弱}

从我国现状来看, 防灾减灾依然由政府主导, 公众将 自然灾害和人为灾害的防范全部依托于政府部门, 公民 灾害风险意识薄弱是全国城乡居民的普遍现象。随着经 济的发展、个人财富的增长, 人们越来越注重生命安 全、生活环境的安全, 安全问题已经成为了不可忽视的 民生诉求。如果不认识到公众灾害风险意识薄弱这一现 状并重视提升社会公众防灾减灾意识, 这将对我国防灾 减灾能力的提升带来不利影响, 一旦灾害发生, 就可能 导致人们的心理危机, 出现哄抢、踩踏、混乱等情况, 严重破坏了社会的稳定，危害国家的发展。

\section{III. 总体国家安全观对防灾减灾的启示}

防灾减灾模式发展至今经历了结构性防灾减灾模 式、非结构性防灾减灾模式、复合防灾减灾模式等过 程, 防灾减灾思路的变化主要是由于灾害的内涵更加丰 富和复杂以及科技的发展带来了防灾减灾的手段的革 新, 然而这些传统防灾减灾模式都或多或少存在弊端。 当前的灾害具有并发性、突发性、异常性等特点, 在传 统防灾减灾模式难以防治各种灾害风险的情况下, 必须 对传统防灾减灾模式进行革新, 构建具有综合性、前瞻 性的全面防灾减灾治理体系。而总体国家安全观作为我 国目前安全工作的总指导, 对我国防灾减灾模式的改 革、全面防灾减灾治理体系的构建提供了重要思路。

从防灾减灾思路上看, 在总体国家安全观被提出之 前, 国家安全主要是指国土安全等传统国家安全，2003 年 “非典” 后, 为了维护社会公共安全, 我国推出 “一 案三制” 灾害管理体系[17], 其中包含了自然灾害、人 
为灾害、公共安全事件等灾害的预防与治理, 是我国内 部安全的内容, 不属于国家安全的范畴。然而在总体国 家安全观提出后，国家安全包含了对自然灾害的管控。

总体国家安全观考虑了现实中传统与非传统安全问题的 串发的实际情况，打破了自然灾害等非传统国家安全问 题和国土安全等传统国家安全问题分开研究治理的界 限, 具有现实意义。如军事冲突中常用导弹打击等方 式，往往伴随着火灾、核泄漏、建筑物倒塌等非传统国 家安全问题的产生。同时，以“9.11” 事件为例，一些 非传统国家安全问题如严重的恐怖袭击也会引发政治安 全问题, 对于具有重大伤害和风险的地震、洪水等自然 灾害, 一般应急管理力量常常无法应对, 甚至需要借助 军事力量的作用。因此, 总体国家安全观将传统安全问 题和非传统安全问题的治理融合起来，前瞻性、系统性 地考虑各种灾害风险并有效进行防灾减灾，以避免顾此 失彼的情况，有利于构建 “防患于未然” 的灾害全面治 理体系，摆脱分开治理的桎梏，形成综合风险治理的观 念和思维。这是总体国家安全观对防灾减灾思路带来的 启示。

从防灾减灾实践上看, 我国成立应急管理部是贯彻总 体国家安全观的一个重要举措。总体国家安全观要求兼 顾对内国家安全和对外国家安全, 并强调不同部门之间 的联动合作。而实现国家对内安全必须要做好应急管理 工作。因此, 为了增强我国应急管理能力, 2018 年 3 月 国务院成立了中国应急管理部，应急管理部有效整合了 各管理部门的职责和资源, 统筹我国应急管理工作, 使 我国应急管理体系更加完善。它的成立是总体国家安全 观下灾害治理体制的伟大创新, 是实现总体国家安全观 目标的重大战略。

\section{IV．总体国家安全观下防灾减灾能力提升策略}

总体国家安全观视域下防灾减灾治理模式强调必须 重视各灾害之间的联系、各部门之间的协调配合、各主 体的共同参与、各环节的系统性预防与应对。综上分 析, 在总体国家安全观下有效提升防灾减灾能力要求: 动员政府、企业、公民社会各类主体的力量，在先进的 综合治理理念的指导下构建防灾减灾制度，将理论与工 程技术相结合, 系统的对传统安全风险和非传统安全风 险进行防范、治理，具体措施有以下几个方面:

\section{A. 多维度构建灾害治理完整框架}

总体国家安全观强调灾害治理的整体性和全面性, 总 体国家安全观下提升防灾减灾能力要求从各个维度构建 灾害治理完整框架[18]。多维度主要体现在以下几点:

\section{1) 整体与局部相结合。}

既要关注地区的灾害风险，使当地政府根据地区的 地理条件、经济水平等实际情况针对性的构建符合当地 特点的灾害治理体系, 又要从总体国家安全层面出发, 制定国家性的灾害治理整体规划和国家安全战略; 同 时, 各级政府职能部门不仅要对该行业的灾害进行风险 防范, 还要相互协调实现灾害治理的整体性功能, 避免 灾害治理中政策的相互分割、缺漏或者交叉，使防灾减 灾资源得到有效利用。为了实现整体与局部的相结合, 在新的国家机构改革中, 我国应急管理部把不同灾害管 理职责部门跨部门加以整合。应急管理部有效的统筹了 分散的灾害治理职责和资源, 极大的提高了灾害应急管
理的效率, 这是我国将自然灾害和事故灾害等主要灾害 的应急职能综合考虑的一个体现, 是总体国家安全观下 的应急体系改革思路, 有利于提高灾害治理框架的协同 性和完整性。

2) 对内安全和对外安全相结合。

气候变暖、恐怖主义、军事冲突等对外安全问题具有 全球性的特点, 很容易引发国内灾害的产生。所以, 总 体国家安全观下构建灾害治理完整框架要求兼顾国内灾 害的防范和国外灾害风险的扩散性和外部性。对此, 应 急管理领域也应延伸，不仅考虑本国范围的应急管理， 还应积极参加国际援救, 实现与其他国家的应急协调合 作。

\section{3) 兼顾有形和无形。}

不仅要对地震、洪水、恐怖袭击等有形灾害进行防范 和治理, 也要关注对经济危机、政治危机等无形灾害。 同时, 既要关注现实的灾害, 又要加强识别和防范可能 会产生灾害的潜在风险或者由灾害带来的各类无形风 险。

\section{B. 扩大全面风险治理对象范围}

总体国家安全体系不仅包含了对内安全, 也包含对外 安全; 即包含了自然安全风险要素, 也包含了人为安全 风险等, 即总体国家安全观几乎考虑了所有安全风险要 素。因此, 总体国家安全观下灾害治理必须要对传统灾 害的概念进行扩展, 突破传统防灾减灾模式中灾种单一 的局限性, 将自然灾害、公共安全事故、恐怖袭击等全 部考虑在内, 将全面风险治理对象扩大到全灾种的范 围, 应包括所有灾害类型, 安全风险要素也应该包括传 统安全风险要素和非传统风险要素, 并注意各灾害之间 的风险关系。要实现风险治理对象的范围的全面性, 可 以通过整合安全治理和应急管理的方式, 这不仅可以提 高应对网络信息灾害、政治灾害等能力, 也保留住自然 灾害等传统灾害应急管理上的优势, 以达到总体国家安 全观全面治理的要求。

\section{C. 加强各阶段的风险治理}

一般将灾害治理过程划分为缩减、预备、反应、恢复 四个阶段[19]。缩减阶段的主要内容是采取措施预防灾 害的产生或者最大限度的削弱灾害带来的影响; 预备阶 段的主要工作是筹备应对灾害所需资源和部署力量, 在 必要时对人员和物资进行及时疏散和转移; 反应阶段的 主要任务是能够在灾害发生时及时制定并执行灾害应对 有效方案。恢复阶段的主要内容是对受灾地区进行灾后 重建以保障该地区的正常生产生活。传统的灾害治理体 系中, 治理灾害的方式、对象和各阶段内容都较为单 一。然而在总体国家安全观视域下防灾减灾治理模式 中, 灾害的内涵更加丰富, 不同灾害之间, 灾害治理的 各个阶段的风险都具有相关性, 对此, 有必要建立各阶 段的风险因子库, 对各阶段各类型的风险进行全面分 析, 以形成系统风险治理体系, 从而提高防灾减灾能力 [20]。

\section{D. 建立以政府为主导, 社会公众广泛参与的灾害治理 主体体系}

目前我国灾害治理的主体主要是政府, 企业和个人在 防灾减灾过程中参与度明显不足, 总体国家安全观下防 
灾减灾能力的提高要求各主体发挥其潜力: 对于政府来 说，政府掌握着灾害治理的大部分资源，能够动员社会 各力量参与到防灾减灾过程中, 可以统筹防灾减灾措施 的实行。对于企业来说, 企业可以在防灾减灾过程中提 供技术支持和资金支持，同时部分企业又是灾害产生的 源头, 既是灾害治理的主体又是灾害治理的客体。因 此, 企业可以从防预灾害和治理灾害两个方面发挥作 用。对于公民来说，虽然个人的力量是有限的，但是公 民具有人口基数大和凝聚力强等特点, 可以在捐赠物 资, 人员救援等方面发挥积少成多的作用。因此, 要提 高全民防灾减灾意识，构建以政府为主导，全社会共同 参与的灾害治理主体体系, 让各个主体在防灾减灾过程 中都能充分发挥自己的作用,

\section{E. 使用多样化灾害治理手段}

传统的防灾减灾模式中, 灾害治理手段较为固定和单 一, 大多是通过技术手段或者管理手段来进行灾害治 理, 但是在总体国家安全观下, 传统国家安全和非传统 国家安全的界限被打破，因此军事手段、外交手段也成 为重要的灾害治理手段, 常见的军事手段有军事打击、 军事演练等，而外交手段有外交谈判，领导人访问等。 同时，灾害的多样化和复杂性使风险识别和分析困难度 增加, 然而在防灾减灾过程中, 风险治理又是必不可少 的内容, 因此, 可以有效利用科学信息技术、人工智能 等手段对风险进行全面分析。

\section{V. 结论}

防灾减灾模式是根据现实灾害治理情况不断完善的, 传统的防灾减灾模式缺乏对灾害的系统考虑和综合性防 灾减灾政策设计，在风险防范方面没有考虑到传统安全 风险因素和非传统安全风险因素的关联性, 在防灾减灾 实践中暴露出防灾减灾体制分割、防灾减灾投入失衡、 重技术轻理念等缺陷。而总体国家安全观丰富了“灾 害” 的内涵, 扩宽了灾害治理的广度和深度, 对防灾减 灾模式的革新具有重要指导性作用。通过总体国家安全 观的启示中，本文探索了全面提升防灾减灾能力的措 施，包括多维度构建灾害治理完整框架、扩大全面风险 治理对象范围、加强各阶段的风险治理、建立以政府为 主导，健全灾害治理主体体系和使用多样化灾害治理手 段。
致谢

本项目受国家自然科学基金项目“西南地区泥石流灾害 损失测度及救灾管理研究”(编号：71661030)资助。

参考文献

[1]谢卓芝, 谢感澜, ““总体国家安全观” 研究综述, ” J. 理论视野, vol. 2016, issue 05, pp. 65-70, 2016.

[2] 陈秋菊, “总体国家安全观背景下社会公共安全应急管理机制研 究,”J. 北京警察学院学报, vol. 2018, issue 03, pp. 34-38, 2018.

[3] 夏文贵, “边境安全问题及其治理,” J. 西北民族大学学报(哲学社 会科学版), vol. 2017, issue 06, pp. 64-70, 2017.

[4] 童星, 陶鹏, “论我国应急管理机制的创新一一基于源头治理、动 态管理、应急处置相结合的理念,”J. 江海学刊, vol. 2013, issue 02, pp. 111-117, 2013.

[5] 李永祥, “什么是灾害? ——灾害的人类学研究核心概念辨析, J 西南民族大学学报 (人文社会科学版), vol. 2011, issue 11, pp. 1220, 2011.

[6] 史培军, “中国综合减灾 25 年: 回顾与展望,” J. 中国减灾, vol 2014, issue 09, pp. 32-35, 2014

[7] 林毓铭, “常态与非常态风险视域的应急管理,” M. 北京: 知识产 出版社, 2015.

[8] 张海波, “应急管理与安全治理:理论趋同与制度整合,” J. 北京行 政学院学报, vol. 2016, issue 01,pp. 1-8, 2016.

[9] 周利敏, “复合型减灾：结构与非结构式困境的破解, ” J. 思想战 线, vol. 2013, issue 06, pp. 76-82, 2013.

[10] 马宝成, “全面践行总体国家安全观着力防范化解重大风险, ” J. 行政管理改革, vol. 2019, issue 04, pp. 17-22, 2019.

[11] 刘杰, 枈维斌, “十八大以来我国公共安全体系建设的理念与实践 创新, J. 福建行政学院学报, vol. 2018, issue 01, pp. 11-18, 2018.

[12] 史培军, 李宁, 叶谦, “全球环境变化与综合灾害风险防范研究,” J. 地球科学进展, vol. 2009, issue 04, pp. 428-435, 2009.

[13] 郭强, “总体国家安全观的理论创新,” J. 人民论坛, vol. 2017, issue 29, pp. 40-41, 2017.

[14] 张海波, “中国总体国家安全观下的安全治理与应急管理, J. 社会 科学文摘, vol. 2016, issue 05, pp. 20-22, 2016.

[15] 李平, 李璐, “建立科学的公共安全管理体制,” J. 北京观察, vol. 2005, issue 12, pp. 63-64, 2005.

[16] 郑功成, “国家综合防灾减灾的现实挑战与战略任务,” J. 中国减灾, vol. 2013, issue 11, pp. 15-17, 2013.

[17] 罗建波, “总体国家安全观与中国国家安全战略,” J. 领导科学论坛, vol. 2018, issue 16, pp. 79-96, 2018.

[18] 刘跃进, “非传统的总体国家安全观,” J. 国际安全研究, vol. 2014 , issue 06, pp. 3-25, 2014.

[19] 薄涛, “我国城市防震减灾能力综合评价研究,”D. 哈尔滨工程大 学, 2009.

[20] 李尧远, 曹蓉“全面风险治理:灾害防治模式的理想形态一一兼论 总体国家安全观的学术启示, ” J. 中国行政管理, vol. 2018, issue 02, pp. 109-113, 2018. 